\title{
Inventário de Habilidades Sociais Educativas do Professor Universitário - Versão Aluno (IHSE-PU-Aluno): Dados Preliminares
}

\author{
Joene Vieira-Santos ${ }^{1,2,3}$, Almir Del Prette, Zilda Aparecida Pereira Del Prette ${ }^{4}$ \\ Universidade Federal de São Carlos, São Carlos-SP, Brasil
}

RESUMO

Este artigo descreve o processo de elaboração do IHSE-PU-Aluno e apresenta os dados obtidos no estudo piloto. A Etapa I desse estudo demonstrou que o instrumento apresenta evidências de validade de conteúdo e que $92,3 \%$ dos itens foram compreendidos sem dúvidas por estudantes universitários. Já a Etapa II demonstrou que vários itens foram sensíveis para verificar a existência de diferenças no repertório de habilidades sociais educativas (HSE) de professores universitários em função de diversas variáveis (sexo do professor, área do conhecimento, primeira graduação, conhecimento prévio do professor, professor avaliado ser supervisor de estágio ou orientador de iniciação de científica ou de monografia do respondente e satisfação do respondente com o desempenho do professor). O IHSE-PU-Aluno pode se mostrar um instrumento valioso para fornecer feedback ao professor sobre sua atuação, bem como para verificar a eficácia de programas de intervenção voltados para o desenvolvimento das HSE desses professores.

Palavras-chave: professor universitário; instrumento; habilidades sociais.

\section{ABSTRACT - Inventory of Educational Social Skills of the University Professor - Student Version (IESS-UP-Student): Preliminary Data}

This paper describes the process of elaborating the IESS-UP-Student (Study 1) and presents the data obtained in the pilot study (Study 2). Study 1 demonstrated that the instrument presents content validity evidence and that $92.3 \%$ of the items were undoubtedly understood by university students. Study 2 showed that several items were sensitive to verify the existence of differences in the repertoire of educational social skills (ESS) of university professors in function of several variables (sex of the professor, area of expertise, first degree, prior knowledge of the professor, the assessed professor being a internship supervisor or mentor in scientific initiation or thesis reviewer, and respondent satisfaction with professor performance). The IESS-UP-Student can prove to be a valuable tool in providing feedback to the professor about their performance, as well as verifying the effectiveness of intervention programs aimed at the development of ESS of these professors.

Keywords: university professor; instrument; social skills.

RESUMEN - Inventario de Habilidades Sociales Educativas del Profesor Universitario - Versión Alumno (IHSE-PUAlumno): Datos Preliminares

Este artículo describe el proceso de elaboración del IHSE-PU-Alumno (Estudio 1) y presenta los datos obtenidos en el estudio piloto (Estudio 2). El Estudio 1 demostró que el instrumento presenta evidencias de validez de contenido y que $92.3 \%$ de los ítems fueron comprendidos sin duda por estudiantes universitarios. Ya el Estudio 2 demostró que varios ítems fueron sensibles para verificar la existencia de diferencias en el repertorio de habilidades sociales educativas (HSE) de profesores universitarios, en función de diversas variables, (sexo del profesor; área de conocimiento; primera graduación; conocimiento previo del profesor; profesor evaluado para ser supervisor de pasantías u orientador de iniciación científica o de monografía del encuestado y satisfacción del mismo con el desempeño del profesor). El IHSE-PU-Alumno puede mostrarse como un instrumento importante para proporcionar feedback al profesor sobre su actuación, así como para verificar la eficacia de programas de intervención destinados al desarrollo de las HSE de esos profesores. Palabras clave: profesor universitario; instrumento; habilidades sociales.

A relação professor-aluno no ensino superior tem sido apontada como uma questão importante da formação, uma vez que seu impacto pode ser observado em diferentes aspectos da experiência acadêmica, tais como adaptação à universidade (Oliveira, Wiles, Fiorin, \& Dias, 2014), interesse e participação em sala (Bariani \& Pavani, 2008), desenvolvimento de valores éticos essenciais ao exercício profissional (Zani \& Nogueira, 2006)

${ }^{1}$ Endereço para correspondência: Laboratório de Interação Social. Grupo de Pesquisa em Relações Interpessoais e Habilidades Sociais. Departamento de Psicologia. Centro de Educação e Ciências Humanas. Universidade Federal de São Carlos. Rodovia Washington Luis, km 235, Caixa Postal 676, 13565-905, São Carlos, SP. Tel.: (16) 3351-8447.E-mail: joenesantos@yahoo.com.br

${ }^{2}$ Agradecimento a Prof. ${ }^{a}$ Dr. ${ }^{a}$ Daniele Lopes e seus alunos pela colaboração na coleta dos dados deste estudo.

3 O presente trabalho foi realizado com apoio da Coordenação de Aperfeiçoamento de Pessoal de Nível Superior - Brasil (CAPES) - Código de Financiamento 001. ${ }^{4}$ Financiamento: CNPq Bolsa Pq1A 306002/2017-5; CNPq Edital Universal 405658/2016-8; Apoio via INCT-ECCE: FAPESP 2014/50909-8; CNPq 465686/2014-1; CAPES 88887.136407/2017-00. 
e competências transversais para o exercício da cidadania (Marinho-Araújo \& Almeida, 2016), permanência na universidade (Bardagi \& Hutz, 2012; Hagenauera \& Volet, 2014), entre outros. No entanto, a qualidade dessa relação envolve diversos aspectos, que vão desde questões relativas ao desempenho do professor e do aluno no ambiente acadêmico até aspectos institucionais (por exemplo, estrutura, normas de conduta indicadas pela instituição de ensino, etc.) (Vieira-Santos \& Henklain, 2017).

Considerando especificamente o desempenho do professor, a qualidade da relação estabelecida com o aluno depende, em grande medida, do repertório de habilidades sociais educativas (HSE) que o docente possui. As HSE referem-se àquelas habilidades sociais "intencionalmente voltadas para a promoção do desenvolvimento e da aprendizagem do outro" (Del Prette \& Del Prette, 2010, p. 95) e o emprego efetivo delas permite ao professor o manejo da situação de ensino-aprendizagem, o que pode resultar em mudanças no repertório comportamental dos alunos (Del Prette \& Del Prette, 2008).

O Inventário de Habilidades Sociais Educativas para Professores Universitários (IHSE-PU), desenvolvido por Del Prette e Del Prette (manuscrito em preparação), avalia o repertório de HSE de docentes universitários. É um instrumento de autorrelato em que o respondente é solicitado a estimar: 1. A frequência com que apresenta o comportamento descrito no item e 2. A dificuldade que experimenta para agir da maneira descrita no item. Os itens do IHSE-PU foram elaborados por Del Prette e Del Prette (manuscrito em preparação), com base em ensaios e pesquisas prévias desses autores sobre ensino e aprendizagem, habilidades sociais dos estudantes como parte da função social da escola e habilidades sociais educativas de professores como requisitos para a consecução de objetivos acadêmicos e socioemocionais da educação escolar (Del Prette \& Del Prette, 1998, 2008; Del Prette \& Del Prette, 2010). Essa proposta vem sendo aplicada à formação do professor do ensino fundamental (Del Prette \& Del Prette, 1997; Del Prette \& Del Prette, 1998) e, mais recentemente, estendida ao ensino superior, em consonância com diversos ensaios sobre esse nível de ensino (por exemplo, Anastasiou, 2012; Pimenta \& Anastasiou, 2005).

A primeira versão do IHSE-PU foi submetida à análise de conteúdo, realizada por especialistas da área de ensino universitário, e análise semântica e de importância dos itens, feita por um grupo de 80 professores universitários. Essas análises resultaram na versão atual do instrumento que se encontra em fase de avaliação de suas propriedades psicométricas.

Como um instrumento de autorrelato, os dados obtidos com o do IHSE-PU expressam a percepção que o professor possui sobre o seu desempenho junto aos alunos. No entanto, a literatura tem apontado que, muitas vezes, a percepção que o professor tem sobre seu desempenho (de uma forma geral) é distinta da percepção de seus aprendizes (Carvalho, 1995; Torelló, 2012; Oliveira et al., 2014). Carvalho (1995), por exemplo, observou que, enquanto os professores diziam agir com autoridade em sala, mais de $50 \%$ dos alunos afirmaram que eles atuavam com autoritarismo e que isso dificultava a comunicação professor-aluno e, consequentemente, o processo de ensino-aprendizagem. Bariani e Pavani (2008) verificaram que, enquanto os estudantes afirmaram que seu interesse e participação em sala eram afetados significativamente pelo desempenho do professor, os docentes atribuíram a participação e o interesse dos alunos ao próprio comportamento do discente. Aparentemente os professores que participaram desse estudo não demonstraram perceber o seu desempenho como um fator significativo que podia afetar a participação do aluno em classe. Assim, as discrepâncias entre a percepção do docente e do discente em relação ao desempenho do professor podem afetar o processo de ensino-aprendizagem, visto que docentes podem supor que a relação estabelecida com os alunos é satisfatória, enquanto os estudantes podem perceber esta relação como coercitiva e dificultadora do processo de aprendizagem. Nesse contexto, instrumentos válidos e confiáveis que permitam comparar a percepção de professores e alunos sobre a atuação do professor podem contribuir para a melhoria dessa atuação e, portanto, do processo de ensino-aprendizagem.

O presente artigo apresenta o Inventário de Habilidades Sociais Educativas do Professor Universitário - versão aluno (IHSE-PU-Aluno), o qual consiste na adaptação do IHSE-PU para ser respondido pelo aluno. A seguir será descrito como se deu o processo de elaboração do IHSE-PU-Aluno, bem como os dados obtidos no estudo piloto.

\section{Método}

O processo de elaboração do IHSE-PU-Aluno envolveu duas etapas. Na Etapa I, foi adaptada uma versão do IHSE-PU a ser respondida pelos alunos, bem como a avaliação dessa versão por especialistas e por estudantes universitários. A Etapa II correspondeu ao estudo piloto. A seguir, serão descritos os procedimentos realizados em cada etapa.

\section{Etapa I - Elaboração do IHSE-PU-Aluno}

Após a autorização dos autores do IHSE-PU, a primeira autora deste artigo reescreveu os itens na perspectiva do aluno e submeteu-os à análise dos autores do instrumento original, que sugeriram pequenas alterações na redação de alguns deles para melhor expressar a ideia contida nos itens originais. Em seguida, os itens adaptados passaram pelos procedimentos de validação de conteúdo e de análise semântica. 
$\mathrm{Na}$ validação de conteúdo, três juízes avaliaram, individualmente, utilizando uma escala do tipo Likert de cinco pontos (de 1, pouquíssimo, a 5, muitíssimo), a clareza da linguagem, a relevância e a representatividade de cada item, considerando as características da população para a qual se destina o instrumento e o conceito de HSE adotado. Além disso, os juízes também foram solicitados a fazer comentários e/ou sugerir alterações nos itens para torná-los mais claros e adequados. Os parâmetros utilizados para a escolha dos juízes foram: (a) desenvolver pesquisas no campo teórico-prático das habilidades sociais e (b) ter experiência na atuação como docente universitário.

As respostas fornecidas pelos juízes permitiram calcular o coeficiente de validade de conteúdo (CVC), conforme propõem Cassepp-Borges, Balbinotti e Teodoro (2010). O cálculo foi realizado para cada um dos itens e para todo o instrumento em cada um dos critérios avaliados: clareza, relevância e representatividade.

A análise semântica foi realizada por duas duplas de estudantes universitários. O procedimento para realizar essa análise consistiu em: (a) apresentar, em linhas gerais, o que são habilidades sociais educativas e o objetivo do instrumento, (b) ler cada item para os participantes e (c) perguntar se o item estava claro. Em caso de resposta negativa, a pesquisadora relia o item. Em caso de resposta afirmativa, a pesquisadora solicitava que os participantes descrevessem em suas próprias palavras o comportamento apresentado no item ou relatassem uma situação ou exemplo que ilustrasse a compreensão que haviam tido do item. Se a compreensão dos alunos estivesse de acordo com o objetivo proposto para o item, a pesquisadora verificava se havia dúvida ou dificuldade com o significado de alguma das palavras que compunham o item. Se não houvesse dúvida, passava-se para o próximo item. Se os alunos tivessem dificuldade com alguma palavra ou expressão, a pesquisadora explicava o sentido e solicitava sugestões para tornar o item mais claro. Se a compreensão dos alunos não estivesse de acordo com o objetivo propostos para o item, a pesquisadora explicava o objetivo do item e solicitava sugestões aos participantes para que o item pudesse ser redigido de maneira mais clara.

\section{Etapa II - Estudo piloto}

\section{Participantes}

Participaram 129 estudantes universitários de instituições públicas de uma cidade de médio porte do interior do estado de São Paulo, sendo 47 homens e $82 \mathrm{mu}-$ lheres, com idade entre 19 e 44 anos $(x=21,8, \pm 3,47$ anos). A amostragem foi por conveniência.

De acordo com a Tabela de Áreas de Conhecimento disponibilizada pela Coordenação de Aperfeiçoamento de Pessoal de Nível Superior (CAPES, 2017), os participantes foram divididos em três grupos em função da graduação que estavam cursando. Os alunos dos cursos de Psicologia, Imagem e Som, Filosofia, Pedagogia, Letras, Ciências Sociais, Linguística e Biblioteconomia compuseram o grupo de ciências humanas. Os alunos das Engenharias (civil, material, química e de produção) e de Gestão Ambiental formaram o grupo de ciências exatas. E, por fim, os alunos dos cursos de Biotecnologia, Biologia, Fisioterapia e Terapia Ocupacional constituíram o grupo de ciências biológicas. A quantidade de respondentes em cada um desses grupos foi, respectivamente, de 56,49 e 24 .

\section{Instrumento}

Versão preliminar do IHSE-PU-Aluno.

\section{Procedimento}

A aplicação do instrumento foi realizada de forma coletiva. O pesquisador entrava em sala de aula (no dia e horário previamente combinados com o professor), explicava os objetivos da pesquisa e convidava os alunos a participar. Na sequência, era solicitado que o participante pensasse em um professor universitário específico com o qual teve aulas e, em seguida, respondesse ao instrumento com base no desempenho desse docente.

O presente estudo faz parte do projeto intitulado Habilidades sociais educativas de docentes universitários: validação de um instrumento e, de acordo com os princípios do Conselho Nacional de Saúde (Resolução ${ }^{\circ}$ 466/12), foi aprovado pelo Comitê de Ética em Pesquisa com Seres Humanos da Universidade Federal de São Carlos (protocolo CAAE: 59553616.6.0000.5504). Todos os participantes que consentiram em participar da pesquisa assinaram o Termo de Consentimento Livre e Esclarecido (TCLE).

\section{Análise de dados}

Os dados obtidos foram analisados e processados utilizando o pacote Statistical Package for the Social Science (SPSS) versão 20. Foram conduzidas análises estatísticas para verificar diferenças nos dados em função das seguintes variáveis: (a) sexo do professor, (b) área do conhecimento, (c) primeira graduação ou não do respondente, (d) conhecimento prévio do professor, ou seja, se o respondente já teve aulas em outras disciplinas e/ou semestre com o professor avaliado, (e) se o professor avaliado é ou foi supervisor de estágio ou orientador de iniciação de científica ou de monografia do respondente, (f) satisfação do respondente com o desempenho do professor, (g) importância atribuída pelo respondente à relação professor-aluno no processo de aprendizagem e (h) avaliação que o respondente faz do seu próprio desempenho acadêmico. Também se verificou o nível de cansaço sentido pelo respondente e a dificuldade/facilidade em manter a concentração até o final do preenchimento do instrumento, bem como as sugestões feitas pelos participantes. 


\section{Resultados e Discussão}

Os resultados e a discussão dos dados coletados serão descritos em etapas. Assim, primeiramente serão apresentados os dados referentes à Etapa I e, em seguida, os da Etapa II.

\section{Etapa I}

A análise de conteúdo por parte dos juízes permitiu calcular o CVC para cada item em cada critério avaliado e o CVC total do instrumento para cada um desses critérios. Como um dos juízes não avaliou o critério de representatividade, os CVCs para cada item e total relacionados a esse critério não foram calculados. A Tabela 1 apresenta a distribuição dos itens em grupos: $\mathrm{CVC} \leq 0,69$, CVC entre 0,70 e 0,79 e $C V C \geq 0,80$.

O CVC total do critério de clareza foi 0,78 , sendo que $83,1 \%$ dos itens apresentaram um $C V C \geq 0,70$. O CVC total do critério de relevância foi de 0,93 , sendo que $100 \%$ dos itens apresentaram CVC $\geq 0,70$. Apesar de Cassepp-Borges et al. (2010) sugerirem que, no processo de tradução cultural de um instrumento, os itens com CVC menor que 0,80 devam ser excluídos, nenhum item foi eliminado nesse momento. Tal decisão foi tomada pelos seguintes motivos: (a) Balbinotti (2004) sugere que CVC superiores a 0,70 podem ser aceitáveis; (b) foram localizados CVC inferiores a 0,70 apenas em relação ao critério de clareza e alterações na redação desses itens poderiam solucionar o problema; (c) não se trata de um processo de tradução cultural, mas sim de adaptação de um instrumento; e (d) o instrumento original ainda encontra-se em fase de validação e, portanto, não foram testados quais são os itens discriminantes do conjunto de itens que atualmente compõem o instrumento.

Tabela 1

Coeficiente de Validade de Conteúdo (CVC) para Cada Item em Cada Critério Avaliado

\begin{tabular}{|c|c|c|c|c|}
\hline & \multicolumn{2}{|l|}{ Clareza } & \multicolumn{2}{|l|}{ Relevância } \\
\hline & Itens & $\%$ & Itens & $\%$ \\
\hline $\mathrm{CVC} \leq 0,69$ & $4,5,6,9,16,20,23,39,58,59$ e 64 & 16,9 & & 0,0 \\
\hline CVC entre 0,70 e 0,79 & $\begin{array}{l}2,8,10,11,14,18,21,24,25,34,35,36 \\
38,40,41,42,43,46,52,55,57 \text { e } 60\end{array}$ & 33,9 & $3,6,34$ e 52 & 6,2 \\
\hline$C V C \geq 0,80$ & $\begin{array}{l}1,3,7,12,13,15,17,19,22,26,27,28 \\
29,30,31,32,33,37,44,45,47,48,49 \\
50,51,53,54,56,61,62,63 \text { e } 65\end{array}$ & 49,2 & $\begin{array}{l}1,2,4,5,7,8,9,10,11,12,13,14,15 \\
16,17,18,19,20,21,22,23,24,25 \\
26,27,28,29,30,31,32,33,35,36 \\
37,38,39,40,41,42,43,44,45,46 \\
47,48,49,50,51,53,54,55,56,57 \\
58,59,60,61,62,63,64 \text { e } 65\end{array}$ & 93,8 \\
\hline
\end{tabular}

O CVC é um dos métodos empregados para avaliar a validade de conteúdo de um instrumento (Pedrosa, Suárez-Álvarez, \& García-Cueto, 2013). Validade de conteúdo, em uma definição mais ampla, refere-se ao grau de relevância e de representatividade que cada elemento de um instrumento demonstra em relação ao construto específico que o teste busca avaliar (Haynes, Richard, \& Kubany, 1995). Pedrosa et al. (2013) definem relevância como o grau em que cada item do instrumento mede o construto e representatividade como a adequação com que o conteúdo do teste representa todas as facetas dele. Os resultados aqui descritos indicam que os itens do IHSE-PU-Aluno mostraram-se adequados para que estudantes universitários avaliem a frequência das HSE de seus professores, visto que $93,8 \%$ dos itens apresentaram CVC $\geq 0,80$ no critério de relevância.

A partir do cálculo dos CVCs e da análise das sugestões/comentários dos juízes, 10 itens foram mantidos sem alteração, 34 itens sofreram pequenas alterações na redação para melhorar a compreensão e 21 itens foram discutidos com os autores do instrumento original. A opção por discutir a alteração desses 21 itens com os autores do instrumento original ocorreu porque as sugestões dos juízes podiam implicar alteração na função de cada item.

Após as modificações descritas acima, os itens foram submetidos à análise semântica. Apenas cinco itens demonstraram alguma dificuldade de compreensão na avaliação dos estudantes universitários. Tais dificuldades foram solucionadas pelo acréscimo de uma palavra ou expressão (nos itens 9, 20 e 29) ou substituição de palavras (nos itens 34 e 54) para torná-los mais claro.

De acordo com Pasquali (2009), a análise semântica é uma etapa importante do processo de elaboração de um instrumento, pois permite verificar se os itens estão sendo compreendidos de maneira adequada pela população a qual se destina o teste. No caso do IHSE-PU-Aluno, $92,3 \%$ dos itens foram compreendidos de maneira adequada pelos estudantes universitários, sem a necessidade de modificações. Os itens que apresentaram algum tipo de dificuldade de compreensão foram modificados de acordo com as sugestões feitas pelos estudantes universitários que participaram da análise semântica. 
A validação de conteúdo e a análise semântica resultaram na versão preliminar do IHSE-PU-Aluno. O instrumento foi composto por três campos: (a) itens adaptados do IHSE-PU, (b) informações pessoais do respondente (no caso, do aluno) e (c) aperfeiçoamento do instrumento. Este último campo destinava-se a obter informações do respondente sobre os níveis de cansaço e dificuldade em manter-se concentrado até o final do preenchimento do instrumento, bem como sugestões, dúvidas e/ou comentários para melhorar o instrumento e/ou sua aplicação.

A versão preliminar do IHSE-PU-Aluno foi analisada pelos autores do instrumento original e por um especialista na área de habilidades sociais para avaliar o layout do instrumento, as instruções e os itens adicionais que visavam coletar informações para a caracterização da amostra. Pequenos ajustes foram feitos para melhorar a redação desses itens adicionais, resultando na versão do IHSE-PU-Aluno que foi aplicada no estudo piloto. Segundo Pedrosa et al. (2013), a validade de conteúdo também envolve as instruções necessárias para a aplicação do instrumento e, neste sentido, submeter a versão preliminar do IHSE-PU-Aluno à avaliação dos autores do IHSE-PU e de um especialista foi importante para aperfeiçoar o instrumento antes de sua aplicação.

\section{Etapa II}

Os dados obtidos com a aplicação do instrumento no estudo piloto foram incialmente tratados para verificar a existência de erros de digitação, valores perdidos no banco de dados e casos atípicos. Não foram identificados erros de digitação, nem casos atípicos. Em relação a valores perdidos, um dos 129 participantes deixou em branco 9,2\% dos itens do instrumento. Apesar de essa ser uma porcentagem aceitável de valores perdidos para um caso (Hair, Black, Babin, Anderson, \& Tatham, 2009), optou-se por excluir os dados desse participante da amostra. Portanto, os dados analisados referem-se às respostas de 128 participantes.

O resultado do teste Kolmogorov-Smirnov (K-S) para uma amostra permitiu verificar que a distribuição das respostas fornecidas pelos participantes a cada item do IHSE-PU-Aluno não diferiu da distribuição normal $(p \leq 0,05)$, permitindo, a priori, o uso de testes paramétricos. A seguir serão discutidos os resultados em função de algumas variáveis.

Em relação à variável sexo do professor, 14 participantes não informaram o sexo do professor avaliado; por isso, para essa análise, foram considerados 114 professores (58 homens e 56 mulheres). Em praticamente todos os itens, o nível de HSE das professoras (feminino) foi maior do que o nível de HSE dos professores (masculino). A única exceção foi o item 3, no qual a média dos professores (masculino) foi maior que a média das professoras (feminino). $\mathrm{O}$ teste $t$ de Student para amostras independentes demonstrou que essa diferença foi estaticamente significante $(p \leq 0,05)$ para $35,4 \%$ dos itens, sendo que o tamanho do efeito $(d)$, de acordo com os parâmetros propostos por Marôco (2011), foi elevado $(d=[0,5 ; 1,0])$ para os itens $13,49,55,57,58$ e 63 , e médio $(d=[0,2 ; 0,5])$ para os itens $2,4,6,14,15,27,28,30$, $31,34,38,42,56,61,62,64$ e 65.

A análise das habilidades sociais envolvidas nos itens que apresentaram diferença estaticamente significativa em função do sexo demonstrou que eles se relacionavam principalmente à comunicação, empatia, expressão de sentimentos positivos, mediação de conflitos, promoção de valores de convivência, promoção de comportamentos de cooperação entre os alunos e solidariedade. Estudos comparando o repertório de habilidades sociais de homens e mulheres têm demonstrado que elas tendem a apresentar um repertório mais elaborado de expressão de sentimentos positivos (Del Prette et al, 2004; Bueno, Oliveira, \& Oliveira, 2001; Bartholomeu, Nunes, \& Machado, 2008), o que pode, em parte, justificar a diferença aqui encontrada.

Também foi verificado se havia diferenças nos dados em função da área de conhecimento (graduação) que o participante cursava. Inicialmente os itens foram examinados para verificar se os dados em cada nível da variável apresentavam uma distribuição normal $(p \leq 0,05)$. Conforme sugere Marôco (2011), aplicou-se o teste de K-S para o grupo de ciências humanas $(n>50)$ e o teste de Shapiro-Wilk para os grupos de ciências exatas e ciências biológicas, pois estes apresentavam $n<50$. Os resultados desses testes demonstraram que alguns itens apresentavam uma distribuição normal $(p \leq 0,05)$ e outros não. No entanto, de acordo com Leech, Barret e Morgan (2005), se o valor de assimetria do item for menor que menos ou mais um $(<+/-1.0)$, a distribuição do item na população pode ser considerada próximo do normal e, portanto, podem-se empregar estatísticas paramétricas. Os itens $21,26,27,30,32,35,38,40,41$ e 57 não atendiam os parâmetros propostos por Leech et al. (2005) para o grupo de ciências exatas ou ciências biológicas e, por isso, a diferença em função da área de conhecimento foi analisado por meio do teste não paramétrico Knuskal-Wallis. Em relação à variância, o teste de Levene demonstrou que ela não era homogênea para os itens 25,50 e 55 , assim, seguindo a orientação de Marôco (2011), a diferença entre as médias desses itens, em função da área de conhecimento, foi analisada por meio do teste F de Welch. Para os demais itens, utilizou-se a ANOVA de um fator para amostras independentes. Foram encontradas diferenças estatisticamente significantes nos itens 25, 32 e 41.

Para o item 25, o teste Welch de $F$ demonstrou que $F_{W}(2,73,094)=3,381, p=0,39$ e $\eta^{2}$ parcial $=0,033$, indicando que a área do conhecimento explicava apenas $3,3 \%$ da variação do item. No entanto, um teste post hoc (Games-Howell) indicou que a diferença foi significativa somente entre os alunos de humanas $(x=1,45, \pm 1,49) \mathrm{e}$ 
biológicas $(x=0,79, \pm 0,93)$. O item 25 referia-se à habilidade do professor de perguntar a opinião dos alunos sobre um determinado assunto, antes de apresentá-lo. É provável que professores de cursos de ciências humanas abordem mais conteúdos que envolvam teorias e argumentos contraditórios (do que professores de cursos da área biológica) e, nessa condição, verificar o conhecimento prévio do aluno pode ser um importante ponto de partida para iniciar a abordagem do assunto. Essa diferença pode justificar, em parte, o resultado encontrado; porém, mais estudos precisam ser realizados para verificar se esse dado se repete em outras amostras.

Para os itens 32 e 41, cujos dados não apresentavam uma distribuição normal, foi aplicado o teste MannWhitney para examinar entre que grupos a diferença era estatisticamente significante $(p \leq 0,05)$. No item 32 , a diferença foi significativa entre os alunos de humanas (posto médio $=44,21$ ) e biológicas (posto médio $=30,35$ ) e entre os alunos de biológicas (posto médio $=40,67$ ) e exatas (posto médio $=29,50$ ). Já no item 41, a diferença foi significativa entre alunos de humanas (posto médio $=43,60)$ e biológicas (posto médio 31,75). Os itens 32 e 41 estavam relacionados à habilidade do professor aproveitar situações de interação com o(s) aluno(s) para abordar questões referentes às relações interpessoais. Assim, o fato dos alunos de humanas atribuírem aos seus professores uma frequência maior nesses itens, quando comparado aos dados dos alunos de exatas e biológicas, pode ter ocorrido porque $78,2 \%$ deles estavam cursando a graduação em Psicologia e esse curso se caracteriza pela discussão de questões desse tipo. No entanto, essa diferença não foi observada no item 19 , que também aborda a discussão de valores e questões de convivência. Apenas novos estudos podem demonstrar se esse é um dado espúrio ou uma relação presente em outras amostras.

A amostra foi composta por 118 participantes que estavam fazendo a primeira graduação, nove já possuíam uma graduação e um não forneceu essa informação (o qual foi excluído das análises para essa variável). Em $81,5 \%$ dos itens, a frequência foi maior quando atribuída por alunos que estavam na primeira graduação do que quando atribuída por alunos que já possuíam uma graduação. O teste $t$ de Student para amostras independentes demonstrou que essa diferença foi estatisticamente significativa $(p \leq 0,05)$ para os itens $58,59,63$ e 64 , nos quais os alunos de primeira graduação atribuíram valores maiores do que alunos que já possuíam uma graduação. O tamanho do efeito da diferença observada nesses itens foi elevado $(d>0,80)$, de acordo com os parâmetros propostos por Marôco (2011).

A análise do conteúdo desses itens demonstrou que todos eles apresentavam situações de interação social que envolvia conflitos e, consequentemente, a apresentação de habilidades sociais assertivas por parte do professor. É possível que alunos que estão cursando uma segunda graduação tenham expectativas mais elevadas sobre o desempenho do professor e, provavelmente, menor tolerância a comportamentos (por parte do docente) que não respeitem os direitos humanos básicos. Assim, ao responderem ao IHSE-PU-Aluno, esses alunos podem ter avaliado o desempenho do professor, em relação a esses itens, de forma mais rigorosa, levando a diferença encontrada. Todavia, apenas novos estudos podem demonstrar se esse é um dado espúrio ou uma relação presente em outras amostras.

Em relação à variável conhecimento prévio do professor, 98 participantes já tinham cursado mais de uma disciplina ministrada pelo professor avaliado, 29 assistiram uma única disciplina do professor avaliado e um respondente não forneceu essa informação. Em todos os itens, a frequência das HSE dos professores com os quais os alunos já haviam tido aulas em outras disciplinas foi maior do que a frequência das HSE atribuída aos professores com os quais os alunos tiveram contato em uma única disciplina. O teste $t$ de Student para amostras independentes demonstrou que essa diferença foi estatisticamente significativa $(p \leq 0,05)$ em $18,5 \%$ dos itens. O tamanho do efeito foi elevado $(d>0,51)$ para os itens 13 , $26,50,55$ e 61 e médio $(0,2<d<0,5)$ para os itens 5,6 , $12,14,19,38$ e 65 .

O exame do conteúdo desses itens permitiu observar que se referem a habilidades mais complexas, tais como automonitoria, mediação de conflitos, monitoria, promoção de cooperação, de valores e de engajamento. O aluno com conhecimento prévio do professor teve mais oportunidades de observar a ocorrência de tais habilidades e, por isso, pode ter tido melhores condições para avaliar a frequência com que tais comportamentos ocorreram.

Quanto ao professor avaliado ser supervisor de estágio ou orientador de iniciação de científica ou de monografia do respondente, apenas 14 participantes avaliaram seu supervisor ou orientador, sendo que destes 10 eram alunos do curso de Psicologia, dois de Biotecnologia, um de Engenharia Química e um de Gestão Ambiental. Em todos os itens, a frequência das HSE desses professores foi maior do que a frequência das HSE dos professores que não tinham desempenhado tais funções junto aos respondentes. O teste $\mathrm{t}$ de Student para amostras independentes demonstrou que essa diferença foi estatisticamente significativa $(p \leq 0,05)$ em $75,4 \%$ dos itens.

A relação mais próxima que se estabelece entre aluno e supervisor - ou orientador -, além de permitir que o aluno tenha um maior conhecimento da maneira de agir do professor, também pode levar a uma atitude mais favorável do aluno para com o professor devido a maior probabilidade de estabelecer uma relação mais afetividade com o docente. Tanto Oliveira-Monteiro e Nunes (2008) como Peixoto, Silvares, Rocha, Monteiro e Pereira (2014) observaram que alunos-estagiários de Psicologia avaliaram o desempenho de seus supervisores de maneira bastante favorável e tiveram dificuldade em 
apontar conflitos ou impasses na relação com o docente. Oliveira-Monteiro e Nunes (2008) sugerem que, essa alta positividade, "para além da percepção de qualidades e/ou virtudes do supervisor, pode embutir negação de conflitos que, se percebidos e/ou manifestados, colocariam o aluno/supervisionando em situação mais vulnerável diante do supervisor do que de um professor" (p. 292). Assim, a frequência mais elevada nas HSE dos supervisores ou orientadores pode ter sido afetada pelo fato de $71,4 \%$ dos respondentes que avaliaram seu supervisor ou orientador eram alunos do curso de Psicologia.

Também se verificou se o fato do aluno ter realizado (ou estar realizando) estágio ou iniciação científica afetava o resultado, visto que um contato mais próximo com professores pode influenciar o nível de exigência do aluno em relação ao desempenho dos professores de uma maneira geral. Apesar de 72,3\% dos itens apresentarem uma média maior quando respondidos por alunos com experiência em estágio ou iniciação científica do que por alunos sem tal experiência, não foram encontradas diferenças estatisticamente significativas $(p \leq 0,05)$ em nenhum dos itens em função do aluno ter experiência com estágio ou iniciação científica.
A variável satisfação do respondente com o desempenho do professor envolveu os seguintes aspectos específicos da relação estabelecida com o professor avaliado: (a) qualidade da relação geral, (b) diálogo com os alunos, (c) respeito que ele demonstra com os alunos, (d) afetividade que ele demonstra pelos alunos, (e) qualidade da atuação docente, (f) o quanto o participante aprende com o professor e (g) disciplina dos alunos nas aulas do professor avaliado. Para cada um deles, o respondente assinalou um valor, numa escala numérica que variava entre -5 e +5 , que representava sua satisfação com aquele aspecto. O teste de KolmogorovSmirnov demonstrou que os dados obtidos em cada um desses aspectos não apresentavam uma distribuição normal, por isso, empregou-se o teste de correlação $\rho$ de Spearman para verificar se havia relação entre cada item e o nível de satisfação em cada aspecto. Para todos os itens, observou-se uma correlação estatisticamente significativa $(p \leq 0,05)$ e positiva com cada um desses aspectos avaliados. A Tabela 2 apresenta o percentual de correlações fracas, moderadas e fortes em cada um dos aspectos, de acordo com os critérios adotados por Dancey e Reidy (2013).

Tabela 2

Percentual de Correlações Fracas, Moderadas e Fortes em Cada um dos Aspectos

\begin{tabular}{|c|c|c|c|c|c|c|}
\hline \multirow{2}{*}{ Aspectos } & \multicolumn{2}{|c|}{ Fraca } & \multicolumn{2}{|c|}{ Moderada } & \multicolumn{2}{|c|}{ Forte } \\
\hline & $N$ & $\%$ & $\mathrm{~N}$ & $\%$ & $\mathrm{~N}$ & $\%$ \\
\hline Qualidade da relação geral & 1 & 1,5 & 47 & 72,3 & 17 & 26,2 \\
\hline Diálogo com os alunos & 1 & 1,5 & 39 & 60,0 & 25 & 38,5 \\
\hline Respeito que ele demonstra com os alunos & 1 & 1,5 & 47 & 72,3 & 17 & 26,2 \\
\hline Afetividade que ele demonstra pelos alunos & 1 & 1,5 & 51 & 78,5 & 13 & 20,0 \\
\hline Qualidade da atuação docente & 0 & 0,0 & 46 & 70,8 & 19 & 29,2 \\
\hline Quanto o participante aprende com o professor & 0 & 0,0 & 51 & 78,5 & 14 & 21,5 \\
\hline Disciplina dos alunos nas aulas do professor avaliado & 4 & 6,2 & 60 & 92,3 & 1 & 1,5 \\
\hline
\end{tabular}

Como é possível perceber, a maioria das correlações entre os itens e a satisfação do aluno com aspectos específicos da relação estabelecida com o professor avaliado foi moderada. A quantidade de correlações fracas foi pequena, sendo que o item 3 apresentou correlações fracas com quase todos os aspectos, com exceção de qualidade da atuação docente e quanto o participante aprende com o professor. Quanto a disciplina dos alunos em sala do professor avaliado, além do item 3 , apresentaram correlações fracas os itens 28, 30 e 53 .

As correlações moderadas e fortes observadas podem sugerir que, quando o professor utiliza as HSE, aumenta o nível de satisfação do aluno com diferentes aspectos do desempenho do docente na relação professor-aluno. Esse resultado parece reproduzir os dados observados em estudos com professores da educação básica, os quais têm demonstrado que, quando o docente desenvolve habilidades sociais, é possível observar mais diálogo e oportunidades de participação ativa dos alunos em sala (Del Prette \& Del Prette, 1997; Del Prette, Del Prette, Torres \& Pontes, 1998) e mais expressões de afeto do professor para com o aluno (Del Prette et al., 1998; Vila, 2005).

Em relação à variável importância atribuída pelo respondente à relação professor-aluno no processo de aprendizagem, o teste de K-S demonstrou que ela não apresentava distribuição normal e, por isso, para correlacioná-la com cada um dos itens, utilizou-se o teste de correlação $\rho$ de Spearman. A correlação foi estatisticamente significativa $(p \leq 0,05)$ apenas para os itens 10,12 , $13,14,19,30,37,38,48,53,54$ e 65 . Todas as correlações foram positivas, porém, fracas $(\rho<0,3)$ de acordo com os parâmetros propostos por Dancey e Reidy (2013). Aparentemente, esse dado sugere que a importância que 
o estudante universitário atribui à relação professor-aluno no processo de aprendizagem não afeta a avaliação que ele faz das HSE do professor.

Por fim, verificou-se diferenças nos dados em função da avaliação que o respondente faz do seu próprio desempenho acadêmico. Para tanto, na seção de informações pessoais do IHSE-PU-Aluno foram incluídas duas questões em que o respondente avaliava, de maneira geral, seu desempenho como aluno. A primeira solicitava que o respondente avaliasse seu aproveitamento geral no curso em termos de notas, assinalando uma das seguintes opções: (a) 10 e 9, (b) 8 e 7, (c) 6 e 5, (d) 4 e 3 ou (e) 2 e 1. Dos 128 participantes, $63,3 \%$ disseram ter aproveitamento entre 8 e 7; 26,6\% entre 6 e 5 e apenas dois afirmaram ter aproveitamento inferior a cinco. A segunda questão solicitava que o respondente avaliasse se julgava o seu desempenho acadêmico displicente/despreocupado ou estudioso/aplicado, marcando um valor em uma escala de zero a 10 , onde zero significava displicente/despreocupado e 10 significava estudioso/aplicado. Dos 128 participantes, $10,9 \%$ marcaram um valor entre 0 a 4 (mais próximo de displicente/despreocupado), $7 \%$ marcaram o ponto central da escala (não se julgava nem displicente nem estudioso) e 82,1\% marcaram um valor entre 6 a 10 (mais próximo de estudioso). Uma vez que a grande maioria dos participantes avaliou seu desempenho de forma positiva em ambas as questões, havendo pouca variabilidade nos dados obtidos com elas, optou-se por não examinar se houve diferenças significativas nas respostas fornecidas aos itens em função dessa variável.

O último grupo de análises realizadas foi em relação às questões que compunham o campo destinado ao aperfeiçoamento do instrumento, as quais procuravam obter informações do respondente sobre os níveis de cansaço e dificuldade em manter-se concentrado até o final do preenchimento do instrumento, bem como sugestões, dúvidas e/ou comentários para melhorar o instrumento e/ou sua aplicação. Em uma escala de zero a 10, onde zero significava "nenhum cansaço" e 10 significava "muitíssimo cansaço" ao responder ao instrumento, $23,4 \%$ dos participantes afirmaram sentir pouco ou nenhum cansaço (assinalaram entre zero e dois); $43,8 \%$ se sentiram cansados (assinalaram entre 3 a 6) e 32,8\% se sentiram muito cansaço (assinalaram entre 7 a 10). O nível de dificuldade/facilidade em manter a concentração também foi avaliado em uma escala de zero a 10, onde zero significava "foi fácil me manter concentrado até o final" e 10 significava "foi difícil me manter concentrado até o final". Nesse aspecto, $35,9 \%$ dos participantes disseram que foi fácil; $27,4 \%$, que não foi fácil nem difícil e $36,6 \%$, que foi difícil manter a concentração. $O$ teste $r$ de Pearson demonstrou que a correlação entre essas duas variáveis é positiva e moderada $(r=+0,50, p=0,001)$.

Do total de 128 participantes, apenas 32 escreveram alguma sugestão e/ou comentário, os quais foram categorizados de acordo com o conteúdo. Os comentários mais frequentes foram: (a) o instrumento é muito extenso e cansativo $(31,2 \%)$, (b) presença de itens redundantes e/ ou cansativos $(25 \%)$, (c) necessidade de uma alternativa para avaliar os itens como "não se aplica" $(15,6 \%)$ e (d) ter uma resposta intermediária entre "raramente" e "muitas vezes" (9,4\%). Entre as categorias de sugestões/ comentários mencionados apenas por um participante, algumas chamaram a atenção: (a) necessidade de ter feito várias disciplinas com o professor para poder responder ao instrumento, (b) dificuldade para compreender o termo "chefe de família" no campo destinado a classificação socioeconômica do respondente, (c) explicitar que o participante imagine o que o professor faria caso não tenha passado pela situação e (d) instrumento tendencioso devido à necessidade do respondente ter que imaginar muitas situações, levando-o a elaborar uma imagem do professor que pode não corresponder à realidade.

Apenas dois participantes fizeram sugestões ou expressaram dúvidas relacionadas a itens específicos. Foram mencionados os itens $3,19,21,35,41$ e 42 , sendo que, destes, apenas os itens 3, 21 e 42 envolviam questões relacionadas à dificuldade de compreensão do seu conteúdo.

A partir dos resultados descritos acima, foram realizadas alterações na estrutura geral do instrumento e em itens específicos. Todas as alterações realizadas foram discutidas com os autores do instrumento original antes de serem efetivadas.

Quanto às mudanças relacionadas à estrutura geral do instrumento, optou-se por:

- Aprimorar as instruções escritas sobre o preenchimento do instrumento. Alguns participantes sugeriram a inclusão da alternativa "não se aplica" (na escala Likert utilizada), pois nunca tinham observado algumas situações descritas nos itens. As novas instruções enfatizam que todas as situações descritas nos itens foram extraídas de observações realizadas em salas de aula e que ocorrem no cotidiano acadêmico.

- Acrescentar a porcentagem ao lado de cada ponto da escala Likert utilizada, pois alguns participantes sugeriram que havia um salto muito grande de "raramente" para "muitas vezes". As porcentagens são um parâmetro a mais para o respondente avaliar, com maior precisão, a frequência com que o professor age conforme descrito no item. A nova escala Likert ficou da seguinte forma: nunca $(0 \%)$, raramente $(25 \%)$, muitas vezes $(50 \%)$, quase sempre $(75 \%)$ e sempre $(100 \%)$.

- Modificar as escalas utilizadas para (1) avaliar a satisfação do aluno com aspectos específicos da relação estabelecida com o professor avaliado e (2) a importância atribuída pelo respondente para a relação professor-aluno, pois ambas possuíam muitos pontos, dificultando o processo de análise dos dados obtidos. Para os dois casos, fez-se a opção por adotar uma escala tipo Likert de cinco pontos. No primeiro caso, a escala foi composta por: muito insatisfeito, insatisfeito, 
indiferente, satisfeito e muito satisfeito. E no segundo variou de "nenhuma importância" a "altíssima importância”.

- Utilizar a versão 2015 do Critério Brasil, pois, no estudo piloto, foi empregada uma versão anterior. Além disso, foi feita uma descrição mais minuciosa sobre como responder a essa parte do instrumento, visto que alguns participantes do estudo piloto ficaram com dúvidas sobre como fazê-lo. Nessa descrição, foi incluída a orientação de que, caso o respondente fosse financeiramente dependente de outra pessoa (por exemplo, pais, avós, tios, etc.), deveria responder aos itens do Critério Brasil de acordo com as quantidades existentes na casa do responsável financeiro. Essas medidas permitirão uma classificação econômica mais precisa dos respondentes.

- Substituir o item período por semestre, pois alguns respondentes, ao invés de colocar o semestre que estavam cursando, colocavam o turno (integral, matutino, vespertino ou noturno).

- Excluir os seguintes itens: (a) "Você fez ou faz estágio ou IC no momento?", (b) aproveitamento geral, (c) avaliação do desempenho acadêmico (displicente/despreocupado - estudioso/aplicado), (d) nível de cansaço sentido pelo respondente, (e) dificuldade/ facilidade em manter a concentração até o final do preenchimento do instrumento e (f) sugestões feitas pelos participantes. Os itens $a, b$ e $c$ foram excluídos porque não demonstraram, a partir das análises estatísticas realizadas, serem significativos. Já os itens $d, e$ e $f$ foram retirados, pois as informações coletadas por meio deles destinavam-se a melhorar a aplicação do instrumento, não se referindo a aspectos importantes do padrão comportamental que o instrumento se destina a avaliar.

Em relação aos itens, dois tipos de alterações foram realizados: modificação do texto ou exclusão do item. O texto dos itens 42,53 e 62 foram modificados para torná-los mais compreensíveis. Já os itens 3, 21 e 52 foram excluídos por diversos motivos. O item 3 (Quando um aluno, ou toda a classe, se comporta de maneira diferente das regras estabelecidas, este professor chama a atenção para tais regras) foi excluído em função (a) da recomendação dos juízes, (b) do CVC abaixo de 0,80 para os critérios de relevância, (c) dos resultados ruins em quase todos os testes estatísticos realizados com os dados do estudo piloto e (d) de ter sido avaliado como de difícil compreensão por um participante no estudo piloto. O item 21 (Se um aluno apresenta dificuldade com algum assunto, o professor coloca alguém da turma para ajudá-lo) foi excluído em função (a) da recomendação dos juízes, (b) do CVC abaixo de 0,80 para os critérios de relevância e clareza, (c) de ter sido avaliado como de difícil compreensão por um participante no estudo piloto e (d) da existência do item 55 que também avaliava o comportamento do professor de mediar a relação entre os alunos. Por fim, a exclusão do item 52 (Se um aluno está encaminhando incorretamente a análise de um conteúdo, o professor faz questionamentos, ajudando-o perceber seus equívocos) foi baseada no CVC abaixo de 0,80 para o critério de relevância e no fato de ser semelhante ao item 36 (Se um aluno apresenta ideias equivocadas, o professor conversa e esclarece, mostrando alternativas para tratar a questão), conforme sinalizado pelos juízes na análise conteúdo. Além disso, o comportamento descrito no item 36 é mais amplo do que o comportamento descrito no item 52, contribuindo para decisão de excluir este e manter aquele.

\section{Considerações finais}

O objetivo deste artigo foi apresentar o processo de elaboração do IHSE-PU-Aluno e os resultados iniciais obtidos na realização do estudo piloto. Os dados da Etapa I desse esse estudo demonstraram que o instrumento apresenta evidências de validade de conteúdo, visto que 93,8\% dos itens apresentaram CVC $\geq 0,80$ no critério de relevância. Além disso, 92,3\% dos itens foram compreendidos sem dúvidas pela população a qual o instrumento se destina, fortalecendo a hipótese de que ele possui uma linguagem clara e compreensível para ser respondido por estudantes universitários.

De acordo com Cassebb-Borges et al. (2010), o estudo piloto consiste em aplicar o instrumento em uma pequena amostra para verificar a adequação dos procedimentos, o nível de compreensão dos itens ou se produz efeitos diferentes dos programados pelo pesquisador. Além disso, os dados do estudo piloto podem auxiliar no processo de decisão sobre a relevância em continuar investigando alguns aspectos do fenômeno estudado (Cozby, 2003). Nesse sentido, a Etapa II foi importante por dois motivos. Primeiramente, demonstrou que vários itens foram sensíveis para verificar a existência de diferenças no repertório de HSE de professores universitários em função de variáveis, como: (a) sexo do professor, (b) área do conhecimento, (c) primeira graduação, (d) conhecimento prévio do professor, (e) professor avaliado ser supervisor de estágio ou orientador de iniciação de científica ou de monografia do respondente e (f) satisfação do respondente com o desempenho do professor. Novas aplicações do instrumento em amostras maiores serão importantes para verificar se os resultados observados se replicam.

Em segundo lugar, a Etapa II permitiu identificar algumas alterações necessárias para tornar o IHSE-PUAluno mais compreensível, resultando no aprimoramento das instruções para o preenchimento do instrumento, bem como na modificação de alguns itens e exclusão de outros. Essas alterações possivelmente contribuirão para que os dados que serão coletados na aplicação posterior do instrumento sejam mais fidedignos e apresentem evidências de validade consistentes. 
O uso das HSE por parte do professor parece estar de acordo com as tendências atuais que compreendem o papel do professor como um mediador do processo de ensino-aprendizagem (Anastasiou, 2012; Masetto, 2003), contribuindo para desenvolver condições que proporcionem ao aluno uma aprendizagem ativa. Nesse sentindo, Del Prette e Del Prette (1998) sugerem que os déficits no repertório de HSE de professores "podem explicar, em parte, o padrão passivo e transmitivo-receptivo ainda predominante nas salas de aula" (p. 211). Dessa forma, o IHSE-PU-Aluno pode se mostrar um instrumento valioso para fornecer feedback ao professor sobre sua atuação, possibilitando a correção de dificuldades na relação professor-aluno e o fortalecimento de padrões de ação que promovem a aprendizagem dos alunos. Além disso, o IHSE-PU-Aluno também poderá ser aplicado juntamente com o IHSE-PU (Del Prette \& Del Prette, manuscrito em preparação), permitindo identificar possíveis discrepâncias entre a percepção que o aluno e que o professor possui do desempenho docente. Tais dados podem ser importantes para planejar intervenções que busquem desenvolver as HSE de docentes universitários, capacitando-os a estabelecer interações mais efetivas e de qualidade com seus alunos. Nesse caso, o IHSE-PU-Aluno também poderá ser um instrumento relevante para avaliar a eficácia de tais intervenções.

De acordo com Hagenauer e Volet (2014), a qualidade da relação estabelecida entre professor e aluno, dentro do ambiente acadêmico, deve ser melhor investigada, pois ela está relacionada: (a) às altas taxas de abandono (quer do ensino superior enquanto sistema, quer de cursos ou de Instituições do Ensino Superior), resultando em custos financeiros e humanos; (b) ao fato de que essa relação afeta os professores e a promoção de um ambiente positivo dentro da sala de aula, também interferem favoravelmente sobre o próprio desempenho docente; e (c) ao aumento de estudos e da importância atribuída à excelência no ensino universitário, o que, segundo os autores, perpassa, entre outros fatores, a relação professor-aluno. Esses autores ainda afirmam que pesquisas futuras deveriam possibilitar o desenvolvimento de uma compreensão mais aprofundada da relação professor-aluno e expandir o entendimento de como as consequências dessa relação afeta alunos e professores. Nessa direção, a elaboração e avaliação das evidências de validade de instrumentos voltadas para aspectos envolvidos na relação professor-aluno (como o IHSE-PU e o IHSE-PU-Aluno) é um passo importante para esse campo de estudo.

\section{Referências}

Anastasiou, L. G. C. (2012). Ensinar, aprender e processos de ensinagem. Em L. G. C. Anastasiou \& L. P. Alves (Eds.), Processos de ensinagem na universidade: pressupostos para as estratégias de trabalho em aula (10a. ed., pp. 15- 43), Joinville, SC: UNIVILLE.

Balbinotti, M. A. A. (2004). Estou testando o que imagino estar? Reflexões acerca da validade dos testes psicológicos. Em C. E. Vaz \& R. L. G. (Eds.), Técnicas projetivas: produtividade em pesquisa. (pp. 22-26), São Paulo: Casa do Psicólogo.

Bardagi, M. P., \& Hutz, C. S. (2012). Rotina acadêmica e relação com colegas e professores: Impacto na evasão universitária. Psico (Porto Alegre), 43(2), 174-184. Recuperado de http://revistaseletronicas.pucrs.br/ojs/index.php/revistapsico/article/view/7870

Bariani, I. C. D., \& Pavani, R. (2008). Sala de aula na universidade: Espaço de relações interpessoais e participação acadêmica. Estudos de Psicologia (Campinas), 25(1), 67-75. doi: 10.1590/S0103-166X2008000100007

Bartholomeu, D., Nunes, C. H. S., \& Machado, A. (2008). Traços de personalidade e habilidades sociais em universitários. Psico-USF, 13(1), 41-50. doi:10.1590/S1413-82712008000100006

Bueno, J. M. H., Oliveira, S. M. S. S., \& Oliveira, J. C. S. (2001). Um estudo correlacional entre habilidades sociais e traços de personalidade. Psico-USF, 6(1), 31-38. doi: 10.1590/S1413-82712001000100005

Carvalho, M. A. V. (1995). Relação professor-aluno: Fatores intervenientes tendo em vista a aprendizagem. Semina: Ciências Sociais e Humanas, 16(2), 57-65. doi: 10.5433/1679-0383.1995v16n3p57

Cassebb-Borges, V., Balbinotti, M. A. A., \& Teodoro, M. L. M. (2010). Tradução e validação de conteúdo: Uma proposta para a adaptação de instrumentos. Em L. Pasquali et al., Instrumentação psicológica: fundamentos e práticas. (pp. 506-520 ) Porto Alegre: Artmed.

Coordenação de Aperfeiçoamento de Pessoal de Nível Superior - CAPES (2017). Tabela de áreas de conhecimento. Brasília, DF. Recuperado de http://www.capes.gov.br/ avaliacao/instrumentos-de-apoio/tabela-de-areas-do-conhecimento-avaliacao

Cozby, P. C. (2003). Métodos de pesquisa em ciência do comportamento. São Paulo: Atlas.

Dancey, C. P., \& Reidy, J. (2013). Estatística sem matemática para psicologia (5 $5^{\mathrm{a}}$ ed.). Porto Alegre: Penso.

Del Prette, A., \& Del Prette, Z. A. P. (2010). Psicologia das relaçôes interpessoais: vivências para o trabalho em grupo (8a ed.). Petrópolis, RJ: Vozes.

Del Prette, A., Del Prette, Z. A. P., Torres, A. C., \& Pontes, A. C. (1998). Efeitos de uma intervenção sobre a topografia das habilidades sociais de professores. Psicologia Escolar e Educacional, 2(1), 11-22. doi: 10.1590/S1413-85571998000100002

Del Prette, Z. A. P., ... Villa, M. B. (2004). Habilidades sociais de estudantes de psicologia: Um estudo multicêntrico. Psicologia: Reflexão e Crítica, 17(3), 341-350. doi: 10.1590/s0102-79722004000300007

Del Prette, Z. A. P., \& Del Prette, A. (1997). Um programa de desenvolvimento de habilidades sociais na formação continuada de professores Em Associação Nacional de Pesquisa Em Educação (Org.). CD-Rom dos trabalhos selecionados para apresentação, (pp. 29). 20a. Reunião Anual da ANPED, Caxambu (MG).

Del Prette, Z. A. P., \& Del Prette, A. (1998). Desenvolvimento interpessoal e educação escolar: O enfoque das habilidades sociais. Temas em Psicologia, 6(3), 217-229. Recuperado de http://pepsic.bvsalud.org/scielo.php?script=sci_arttext\&pid=S1413-389X1998000300005 
Del Prette, Z. A. P., \& Del Prette, A. (2008). Um sistema de categorias de habilidades sociais educativas. Paidéia, 18(41), 517-530. doi: 10.1590/S0103-863X2008000300008

Del Prette, Z. A. P., \& Del Prette, A. (manuscrito em preparação). Inventário de Habilidades Sociais Educativas - Cersão Professor Universitário (IHSE-PROF-UNIV): Estudos psicométricos em andamento.

Del Prette, Z. A. P., Del Prette, A., Garcia, F. A., Silva, A. T. B., \& Puntel, L. P. (1998). Habilidades sociais do professor em sala de aula: Um estudo de caso. Psicologia: Reflexão e Crítica, 11(3), 591-603. doi:10.1590/S0102-79721998000300016

Haynes, S. N., Richard, D. C. S., \& Kubay, E. S. (1995). Content validity in psychological assessment: A functional approach to concepts and methods. Psychological Assessment, 7(3), 238-247. doi: 10.1037/1040-3590.7.3.238

Hair, J. F., Black, W. C., Babin, B. J., Anderson, R. E., \& Tatham, R. L. (2009). Análise multivariada dos dados (6 ed.). Porto Alegre: Bookman.

Hagenauer, G., \& Volet, S. E. (2014). Teacher-student relationship at university: An important yet under-researched field. Oxford Review of Education, 40(3), 370-388. doi: 10.1080/03054985.2014.921613

Leech, N., Barret, K., \& Morgan, G. A. (2005). SPSS for intermediate statistics: Use and interpretation (2 $2^{\mathrm{a}}$ ed.). New Jersey: Lawrence Erlbaum Associates.

Marinho-Araújo, C., \& Almeida, L. (2016). Abordagem de competências, desenvolvimento humano e educação superior. Psicologia: Teoria e Pesquisa, 32(5), 1-10. doi:10.1590/0102-3772e32ne212

Marôco, J. (2011). Análise estatística com SPSS Statistics (5 $5^{\mathrm{a}}$ ed.). Lisboa: Edições Sílabo.

Masetto, M. T. (2003). Competência pedagógica do professor universitário. São Paulo: Summus.

Oliveira, C. T., Wiles, J. M., Fiorin, P. C., \& Dias, A. C. G. (2014). Percepções de estudantes universitários sobre a relação professor-aluno. Psicologia Escolar e Educacional, 18(2), 239-246. doi: 10.1590/2175-3539/2014/0182739

Oliveira-Monteiro, N. R., \& Nunes, M. L. T. (2008). Supervisor de psicologia clínica: Um professor idealizado? Psico-USF, 13(2), $287-296$. Recuperado de http://pepsic.bvsalud.org/scielo.php?script=sci_arttext\&pid=S1413-82712008000200015

Pasquali, L. (2009). Psicometria: Teoria dos testes na Psicologia e na Educação (3 ${ }^{\mathrm{a}}$ ed.). Petrópolis, RJ: Vozes.

Pedrosa, I., Suárez-Álvarez, J., \& García-Cueto, E. (2013). Evidencias sobre la validez de contenido: Avances teóricos y métodos para su estimación. Acción Psicológica, 10(2), 3-18. doi: 10.5944/ap.10.2.11820

Peixoto, A. C. A., Silvares, E. F. M., Rocha, M. M., Monteiro, N. R. O., \& Pereira, R. F. (2014). A percepção de estagiários em diferentes IES do Brasil sobre a supervisão. Psicologia: Ciência e Profissão, 34(3), 528-539. doi: 10.1590/1982-3703001482013

Pimenta, S. G., \& Anastasiou, L. G. (2005). Docência no Ensino Superior (2 ed.). São Paulo: Cortez.

Vieira-Santos, J., \& Henklain, M. (2017). Contingências sociais que dificultam o engajamento do professor universitário em relações de qualidade com seus alunos. Revista Perspectivas em Análise do Comportamento, 8(2), 200-214. doi: 10.18761/pac.2016.032

Vila, E. M. (2005). Treinamento de Habilidades Sociais em grupo com professores de crianças com dificuldades de aprendizagem: Uma análise sobre procedimentos e efeitos da intervenção (Dissertação de mestrado), Universidade Federal de São Carlos, São Paulo, Brasil.

Torelló, O. M. (2012). Las competencias del docente universitario: la percepción del alumno, de los expertos y del propio protagonista. Revista de Docencia Universitaria, 10(2), 299-318. doi: 10.4995/redu.2012.6109

Zani, A. V., \& Nogueira, M. S. (2006). Incidentes críticos do processo ensino-aprendizagem do curso de graduação em enfermagem, segundo a percepção de alunos e docentes. Revista Latino-Americana de Enfermagem, 14(5), 742-748. Recuperado de http://www.redalyc. org/articulo.oa?id=281421864016

\section{Sobre os autores}

Joene Vieira-Santos é psicóloga, Mestre em Teoria e Pesquisa do Comportamento (pela UFPA) e doutoranda no Programa de PósGraduação em Psicologia da Universidade Federal de São Carlos (UFSCar). Bolsista CAPES.

Almir Del Prette é psicólogo, doutor em Psicologia (Psicologia Experimental) pela Universidade de São Paulo (1990). Atualmente é Professor Titular Sênior (Voluntário) vinculado aos Programa de Pós-Graduação em Educação Especial e Pós-Graduação em Psicologia da Universidade Federal de São Carlos.

Zilda Aparecida Pereira Del Prette é psicóloga, com pós-doutorado em Psicologia das Habilidades Sociais na Universidade da Califórnia (2002). Atualmente é pesquisadora nível IA do CNPq, Professora Titular Sênior da Universidade Federal de São Carlos, vinculada ao Programa de Pós-Graduação em Psicologia da Universidade Federal de São Carlos e ao Instituto Nacional de Ciência e Tecnologia em Comportamento, Cognição e Ensino (INCT-ECCE). 